\title{
Mutation screening of the SLC26A4 gene in a cohort of 192 Chinese patients with congenital hypothyroidism
}

Chunyun Fu' ${ }^{*}$, Haiyang Zheng ${ }^{1 *}$, Shujie Zhang' ${ }^{\prime}$ Yun Chen', Jiasun Su', Jin Wang', Bobo Xie', Xuyun Hu', Xin Fan', Jingsi Luo', Chuan Li', Rongyu Chen', Yiping Shen', ${ }^{1,2}$ Shaoke Chen'

\begin{abstract}
Objective: Pendred syndrome (PS) is an autosomal recessive disorder characterised by sensorineural hearing loss and thyroid dyshormonogenesis. It is caused by biallelic mutations in the SLC26A4 gene encoding for pendrin. Hypothyroidism in PS can be present from birth and therefore diagnosed by neonatal screening. The aim of this study was to examine the SLC26A4 mutation spectrum and prevalence among congenital hypothyroidism $(\mathrm{CH})$ patients in the Guangxi Zhuang Autonomous Region of China and to establish how frequently PS causes hearing impairment in our patients with $\mathrm{CH}$. Subjects and methods: Blood samples were collected from $192 \mathrm{CH}$ patients in Guangxi Zhuang Autonomous Region, China, and genomic DNA was extracted from peripheral blood leukocytes. All exons of the SLC26A4 gene together with their exon-intron boundaries were screened by nextgeneration sequencing. Patients with SLC26A4 mutations underwent a complete audiological evaluation including otoscopic examination, audiometry and morphological evaluation of the inner ear. Results: Next generation sequencing analysis of SLC26A4 in $192 \mathrm{CH}$ patients revealed five different heterozygous variations in eight individuals (8/192, 4\%). The prevalence of SLC26A4 mutations was $4 \%$ among studied Chinese $\mathrm{CH}$. Three of the eight were diagnosed as enlargement of the vestibular aqueduct (EVA), no PS were found in our $192 \mathrm{CH}$ patients. The mutations included one novel missense variant p.P469S, as well as four known missense variants, namely p.V233L, p.M147I, p.V609G and p.D661E. Of the eight patients identified with SLC26A4 variations in our study, seven patients showed normal size/location of thyroid gland, and one patients showed a decreased size one. Conclusions: The prevalence of SLC26A4 pathogenic variants was $4 \%$ among studied Chinese patients with $\mathrm{CH}$. Our study expanded the SLC26A4 mutation spectrum, provided the best estimation of SLC26A4 mutation rate for Chinese $\mathrm{CH}$ patients and indicated the rarity of PS as a cause of CH. Arch Endocrinol Metab. 2016;60(4):323-7
\end{abstract}

${ }^{1}$ Department of Genetic Metabolism, Children's Hospital, Maternal and Child Health Hospital of Guangxi Zhuang Autonomous Region, Nanning, People's Republic of China 2'Boston Children's Hospital, Harvard Medical School, Boston, MA, United States

* Chunyun Fu and Haiyang Zheng contribute equally to this work

Correspondence to:

Shaoke Chen

Department of Genetic Metabolism,

Children's Hospital, Maternal and

Child Health Hospital of Guangxi

Zhuang Autonomous Region, Nanning

530012 - Guangxi, P. R. China

chenshaoke123@163.com

Received on July/13/2015 Accepted on Aug/19/2015

DOI: 10.1590/2359-3997000000108

\section{INTRODUCTION}

$\mathrm{P}$ endred syndrome(PS) is an autosomal recessive disease, characterised by functional impairment of the thyroid gland due to thyroid dyshormonogenesis, sensorineural hearing loss, and developmental malformations of the inner ear $(1,2)$. In about $30 \%$ of patients dyshormonogenesis is present at birth and is diagnosed by neonatal screening for congenital hypothyroidism $(\mathrm{CH})$. It is caused by homozygous or compound heterozygous mutations in the SLC26A4 gene encoding pendrin, a multifunctional anion exchanger that is highly expressed in the thyroid, the inner ear and the kidneys (3-5).

In the thyroid, pendrin is expressed at the apical surface of thyrocytes. It acts as a chloride-iodide exchanger transporting iodide from the cell to the colloid in the follicular lumen, where iodide is organified (68). Defect in SLC26A4 cause loss of pendrin function, which results in defective iodide organification that induces thyroid overgrowth and goiter in most affected individuals (9-11). However, the phenotypes are variable among different individuals. Up to now, the mutational spectrum of the SLC26A4 and the genotype-phenotype relationships has not yet been fully established, no study has been designed to assess its prevalence among Chinese $\mathrm{CH}$ patients, and very little is known about the proportion of patients with PS among children with $\mathrm{CH}$. Our study therefore aimed to ascertain patients carrying mutations in the SLC26A4 gene among subjects with $\mathrm{CH}$. We conducted this study with two main goals: 
(1) to examine the $S L C 26 A 4$ mutation spectrum and prevalence among patients with $\mathrm{CH}$ in Guangxi Zhuang Autonomous Region, and (2) to assess the incidence of PS in a cohort of subjects with $\mathrm{CH}$.

\section{SUBJECTS AND METHODS}

\section{Subjects}

We enrolled $192 \mathrm{CH}$ patients (101 females and 91 males), who were identified through newborn screening among 623,000 newborns in the Guangxi Zhuang Autonomous Region, China, from October 2010 to June 2014. Newborn screening was done with filter paper for $\mathrm{CH}$ between $72 \mathrm{~h}$ and 7 days after birth, blood samples were collected from the heel and TSH level was measured by time-resolved fluorescence assay. Subjects with increased TSH (TSH $\geq 8 \mathrm{mIU} / \mathrm{l}$ ) levels observed during neonatal screening were recalled for further evaluation. Serum TSH and FT4 were determined by electrochemiluminescence assay. Diagnosis of $\mathrm{CH}$ is based on elevated TSH levels (TSH $\geq 10 \mathrm{mIU} / \mathrm{l}$ ) and decreased FT4 levels (FT4 < 12 pmol/l). Thyroid ultrasonography was performed during the neonatal period before treatment. The glands outside reference ranges of newborns in our population (right lobe: 1.44 $\pm 0.22 \mathrm{~cm}$ in length, $0.72 \pm 0.11 \mathrm{~cm}$ in width and $0.63 \pm 0.09 \mathrm{~cm}$ in thickness; left: $1.44 \pm 0.21 \mathrm{~cm}$ in length , $0.70 \pm 0.10 \mathrm{~cm}$ in width and $0.63 \pm 0.09 \mathrm{~cm}$ in thickness) were regarded as decreased or enlarged (12). The study was approved by the local Medical Ethics Committee. Informed consent was obtained from the parents of the patients.

\section{Mutation detection and audiological evaluation}

Peripheral venous blood samples were collected from the patients. Genomic DNA was extracted from peripheral blood leukocytes using QIAamp DNA Blood Mini Kit (Qiagen, Germany) according to the manufacturer's protocol. All exons of SLC26A4 with their flanking intronic regions were amplified in a $50 \mu \mathrm{L}$ reaction mixture containing 100-250 ng of genomic DNA, $1 \times$ Taq Buffer with $50 \mathrm{mM}$ of $\mathrm{KCl}$, $2.5 \mathrm{mM}$ of $\mathrm{MgCl}, 200 \mu \mathrm{M}$ of $\mathrm{dNTP}, 1$ unit of Taq DNA polymerase (Fermentas, USA), and 20 pmol of forward and reverse primers were prepared. Thirty cycles of amplification were carried out with a standard PCR protocol. PCR products were purified using QIA quick PCR purification kit (Qiagen, Germany) following the manufacturer's instructions. The purified PCR products were sequenced using Illumina MiSeq (Illumina, USA). The Illumina Amplicon Viewer was used for data analysis, and the SnpEff was used for variant annotation. Patients with SLC26A4 mutations underwent a complete audiological evaluation including otoscopic examination and audiometry. Morphological evaluation of the inner ear was investigated by highresolution $\mathrm{CT}$ of temporal bones in coronal and axial planes. Enlargement of the vestibular aqueduct (EVA) was defined as a vestibular aqueduct diameter exceeding $1.5 \mathrm{~mm}$ at the midpoint between the posterior cranial fossa and the vestibule of the inner ear.

\section{RESULTS}

Next generation sequencing analysis of SLC26A4 in $192 \mathrm{CH}$ patients revealed five different heterozygous variations in eight individuals $(8 / 192,4 \%)$. The prevalence of $S L C 26 A 4$ variants was $4 \%$ among studied Chinese patients with $\mathrm{CH}$. The variants included one novel missense variant p.P469S, as well as four known missense mutations, namely p.V233L, p.M147I, p.V609G and p.D661E. All variants were confirmed by Sanger sequencing (Figure 1). Polyphen and Mutation taster predicted that the novel variation would have deleterious effects, by damaging the SLC26A4 protein. Additionally, the variant was not seen in normal population in the database of dbSNP, ESP or 1000 genomes. DNAMAN software was then used to carry out multiple sequence alignment of the SLC26A4 family protein from the following species: Homo sapiens, Mus musculus, Rattus norvegicus, Canis lupus familiaris and Sus scrofa. The p.P469S variation was found to be located in highly conserved region of SLC26A4. Those all suggesting that the amino acid substitution might be pathologic mutation.

The clinical features and laboratory test results are summarized in the Table 1 . All patients were born at fullterm to unrelated parents and diagnosed with $\mathrm{CH}$ by newborn screening. All patients with SLC26A4 variants underwent a complete audiological evaluation. Three of eight patients were diagnosed as EVA with moderate to severe hearing problems. As for thyroid morphology, seven of the eight patients showed normal size/location of thyroid gland. Ultrasonography showed Patient 7 with decreased size of thyroid gland (right lobe: 1.1 $\times 0.6 \times 0.5 \mathrm{~cm}$; left: $1.1 \times 0.6 \times 0.5 \mathrm{~cm})$. No PS was diagnosed in our cohort of $192 \mathrm{CH}$ patients. 
P1
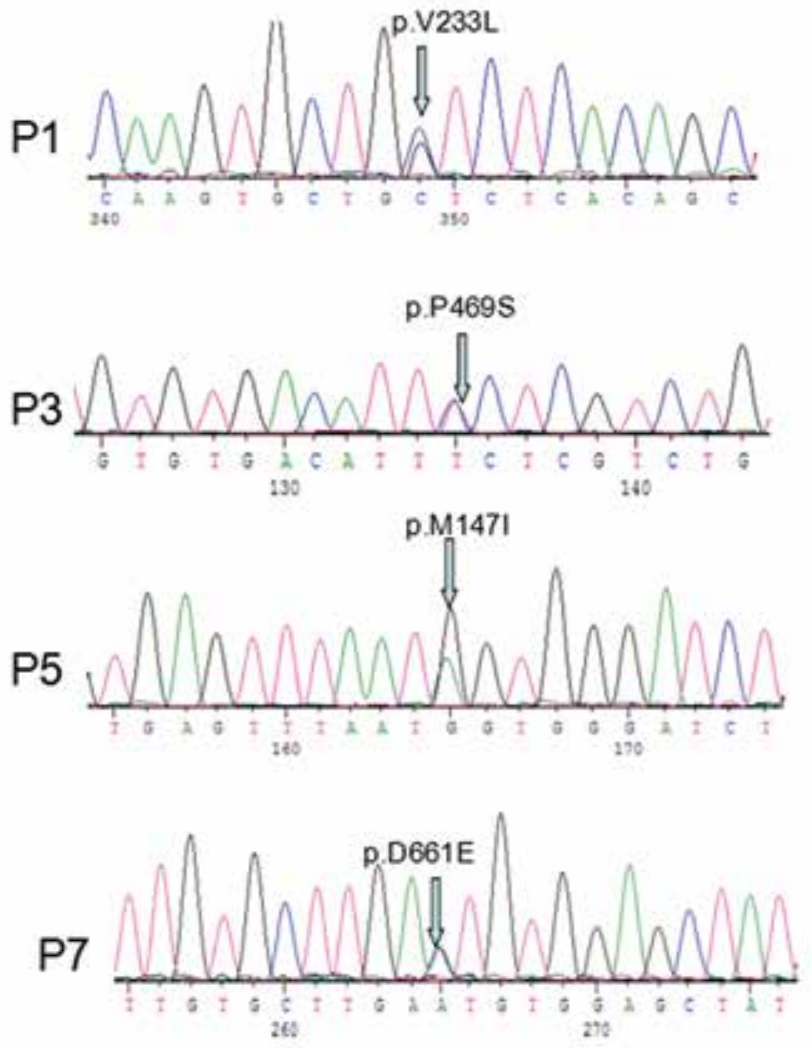

P2

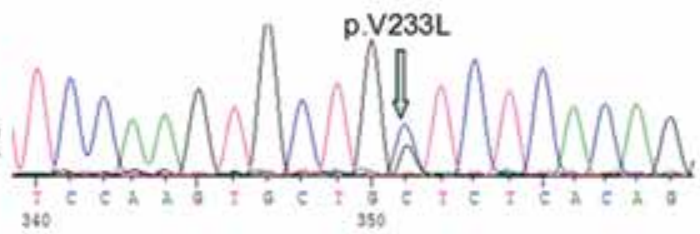

P4
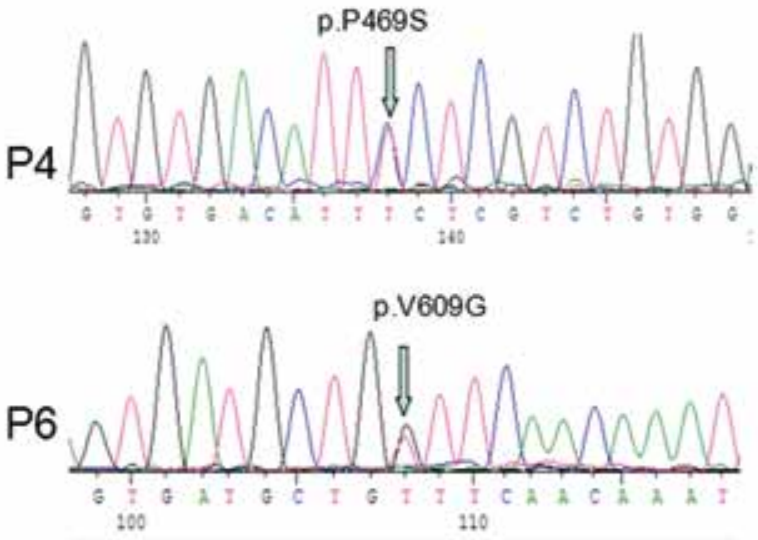

P8

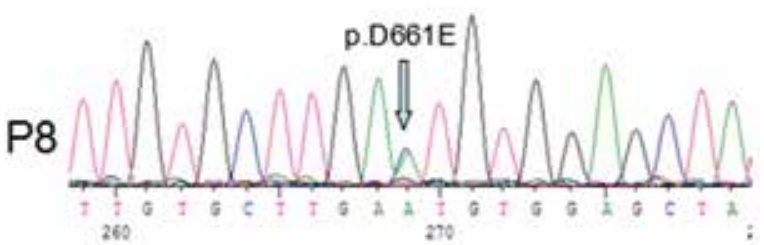

Figure 1. Mutations in SLC26A4 in patients with $\mathrm{CH}$. Figures (P1-P8) showed different sequences in identified eight affected individuals. P1, P2 showed the same SLC26A4 heterozygous mutation p.V233L. P3, P4 showed the same SLC26A4 heterozygous mutation p.P469S. P5, P6 showed heterozygous mutations p.M147I and p.V609G, respectively. P7, P8 showed the same SLC26A4 heterozygous mutation p.D661E.

Table 1. Clinical features, laboratory results and SLC26A4 gene mutations in eight patients

\begin{tabular}{|c|c|c|c|c|c|c|c|c|c|c|}
\hline Patient & Sex & $\begin{array}{l}\text { Age* }^{*} \\
\text { (year) }\end{array}$ & $\begin{array}{l}\text { Weight }{ }^{\star}(\mathbf{k g}) \\
\text { centile }\end{array}$ & $\begin{array}{l}\text { Length* }(\mathrm{cm}) \\
\text { centile }\end{array}$ & $\begin{array}{l}\mathrm{TSH}^{\star} \\
\text { (mlU/l) }\end{array}$ & $\begin{array}{l}\mathrm{FT4}^{\star} \\
(\mathrm{pmol} / \mathrm{l})\end{array}$ & $\begin{array}{l}\text { Initial dose of } \\
\text { L-T4( } \mu \mathrm{g} / \mathrm{kg} / \\
\text { day) }\end{array}$ & $\begin{array}{l}\text { Thyroid } \\
\text { morphology }\end{array}$ & $\begin{array}{c}\text { SLC26A4 } \\
\text { variations }\end{array}$ & $\begin{array}{c}\text { Clinical } \\
\text { phenotype }\end{array}$ \\
\hline 1 & Male & NS & $\begin{array}{c}3 \\
\left(10^{\text {th }}-25^{\text {th }}\right)\end{array}$ & $\begin{array}{c}50 \\
\left(25^{\left.5^{\mathrm{th}}-50^{\text {th }}\right)}\right.\end{array}$ & 43 & 10 & 6.3 & Normal & p.V233L(het) & $\mathrm{CH}+\mathrm{EVA}$ \\
\hline 2 & Female & NS & $\begin{array}{c}3.2 \\
\left(25^{\text {th}}-5^{\text {th }}\right)\end{array}$ & $\begin{array}{c}50 \\
\left(50^{\left.\text {th }-75^{\text {th }}\right)}\right.\end{array}$ & $>100$ & 3.67 & 11.6 & Normal & p.V233L(het) & $\mathrm{CH}$ \\
\hline 3 & Male & NS & $\begin{array}{c}3.8 \\
\left(75^{\text {th }}-90^{\text {th }}\right)\end{array}$ & $\begin{array}{c}51 \\
\left(50^{\text {th- }}-75^{\text {th }}\right)\end{array}$ & $>100$ & 5.57 & 8.6 & Normal & p.P469S(het) & $\mathrm{CH}+\mathrm{EVA}$ \\
\hline 4 & Male & NS & $\begin{array}{c}3.45 \\
\left(50^{\text {th }}-75^{\text {th }}\right)\end{array}$ & $\begin{array}{c}50 \\
\left(25^{\text {th }}-50^{\text {th }}\right)\end{array}$ & $>100$ & 2.22 & 13.5 & Normal & p.P469S(het) & $\mathrm{CH}+\mathrm{EVA}$ \\
\hline 5 & Male & NS & $\begin{array}{c}3.15 \\
\left(25^{\text {th }}-50^{\text {th }}\right)\end{array}$ & $\begin{array}{c}50 \\
\left(25^{\text {th }}-50^{\text {th }}\right)\end{array}$ & $>100$ & 2.41 & 9.0 & Normal & p.M147l(het) & $\mathrm{CH}$ \\
\hline 6 & Female & NS & $\begin{array}{c}2.7 \\
\left(3^{\text {th }}-10^{\text {th }}\right)\end{array}$ & $\begin{array}{c}49 \\
\left(25^{t^{\mathrm{th}}}-50^{\text {th }}\right)\end{array}$ & $>100$ & 8.5 & 13.0 & Normal & p.V609G(het) & $\mathrm{CH}$ \\
\hline 7 & Female & NS & $\begin{array}{c}3.02 \\
\left(25^{\left.\text {th }^{\mathrm{t}}-50^{\text {th }}\right)}\right.\end{array}$ & $\begin{array}{c}50 \\
\left(50^{\text {th }-75^{\text {th }}}\right)\end{array}$ & $>100$ & 1.13 & 12.8 & Decreased & p.D661E(het) & $\mathrm{CH}$ \\
\hline 8 & Male & NS & $\begin{array}{c}3.1 \\
\left(25^{\text {th }}-50^{\text {th }}\right)\end{array}$ & $\begin{array}{c}50 \\
\left(25^{\text {th }}-50^{\text {th }}\right)\end{array}$ & 11 & 10 & 6.0 & Normal & p.D661E(het) & $\mathrm{CH}$ \\
\hline
\end{tabular}

${ }^{*}$ Age, weight, length, TSH, FT4 at diagnosis.

NS: newborn screening. 


\section{DISCUSSION}

$\mathrm{CH}$ is a common endocrine disorder with prevalence ranging from $1: 2000$ to $1: 4000$ newborns $(13,14)$. Iodine deficiency is still the major cause of neonate hypothyroidism worldwide $(15,16)$, and the prevalence was reported to be $2.09 \%$ in Guangxi Zhuang Autonomous Region (17). Apart from iodine deficiency, the sporadic $\mathrm{CH}$ cases can be caused by mutations in a variety of genes including $\operatorname{SLC} 26 A 4(13,18)$.

In this study, we conducted the largest SLC26 A4 gene mutation screening so far in $\mathrm{CH}$ patients. All the patients were initially identified by newborn screening and subsequently confirmed with re-evaluation. The result of our study revealed five different heterozygous variations in eight individuals $(8 / 192,4 \%)$. The prevalence of SLC26A4 mutations was $4 \%$ among $\mathrm{CH}$ patients in Guangxi Zhuang Autonomous Region, China.

PS is an autosomal recessive disorder which is caused by biallelic mutations in the SLC26A4 gene. The SLC26A4 mutation leads to the development of a variable clinical spectrum of hearing loss due to inner-ear malformation as an EVA or mondini cochlea associated with goiter and in some cases $\mathrm{CH}$. A combination of three parameters was suggested to define and diagnose PS $(11,19)$ : (i) sensorineural deafness with bilateral EVA; (ii) thyroid abnormality comprising goiter and/ or hypothyroidism and/or a positive perchlorate discharge test; (iii) biallelic SLC26A4 mutations. In our study, we found eight patients with $\mathrm{CH}$ had monoallelic SLC26A4 mutations and three of them had EVA. No PS was diagnosed in our $192 \mathrm{CH}$ patients. Up to now, the pathogenic role of monoallelic SLC26A4 mutations remains unclear. Although both biallelic and monoallelic SLC26A4 mutations were associated with EVA, we cannot exclude the possibility that the second allele of the SLC26A4 gene was carrying a mutation, which would not have been identified if located in an intronic or regulatory sequence.

In the same way, patients with monoallelic SLC26A4 mutations have a very low risk for developing a $\mathrm{CH}$. However, we cannot exclude the possibility that the second mutation has not been identified. Or it was caused by other $\mathrm{CH}$ associated gene mutations or environmental factors.

PS is characterized by sensorineural deafness and goiter. Goiter was part of the initial description of PS but is not now considered a constant feature (20-22). In countries with a high iodine intake, goiter development and thyroid dysfunction are usually not seen in patients with biallelic mutations in the SLC26A4 gene (11). In some cases, thyroid hypoplasia can also be found in patients with biallelic SLC26A4 mutations (23). In this study, thyroid ultrasound showed seven of the eight patients with SLC26A4 heterozygous variations had normal size/location of thyroid gland, and the other one had decreased thyroid gland.

Up to now, the SLC26A4 genotype-phenotype relationships has not yet been fully established, the variability of the phenotypes were observed in different mutations of SLC26A4, even in the same mutation among members of the same family. In our study, patients 1-2 shared the same heterozygous mutation (p.V233L), but the clinical phenotypes such as TSH levels, hearing test varied greatly. Variable phenotypes are presumed to be caused by 1) other genetic factors such as gene mutations or modifier genes; 2) individual differences or stochastic phenomena, and 3) environmental factors (iodine uptake, nutrition etc.).

In conclusion, we conducted the largest SLC26A4 mutation screening for a cohort of patients with $\mathrm{CH}$ in Guangxi Zhuang Autonomous Region, China. We identified SLC26A4 heterozygous pathogenic variant in eight of 192 cases $(4 \%)$. Three of the eight were diagnosed as EVA, no PS were found in our $192 \mathrm{CH}$ patients. our results indicate the rarity of PS as a cause of $\mathrm{CH}$. We reported one novel SLC26A4 pathogenic variant (p.P469S), our study expanded the SLC26A4 mutation spectrum and provided the best estimation of SLC26A4 mutation rate for Chinese $\mathrm{CH}$ patients.

Acknowledgments: this study was supported by the National Natural Science Foundation of China (81260126), Key Projects of Guangxi Health Department (2012025) and Guangxi Natural Science Foundation Program (2012GXNSFAA053174).

Disclosure: no potential conflict of interest relevant to this article was reported.

\section{REFERENCES}

1. Park MK, Sagong B, Lee JD, Bae SH, Lee B, Choi KS, et al. A1555G homoplasmic mutation from A1555G heteroplasmic mother with Pendred syndrome. Int J Pediatr Otorhinolaryngol. 2014;78(11):1996-9.

2. Huang CJ, LeiTH, Chang WL, TuTY, Shiao AS, Chiu CY, et al. A Novel mutation in the SLC26A4 gene in a Chinese family with Pendred syndrome. Int J Pediatr Otorhinolaryngol. 2013;77(9):1495-9.

3. Chen J, Wei Q, Yao J, Qian X, Dai Y, Yang Y, et al. Identification of two heterozygous deafness mutations in SLC26A4 (PDS) in a Chinese family with two siblings. Int J Audiol. 2013;52(2):134-8.

4. Sagong B, Seok JH, Kwon TJ, Kim UK, Lee SH, Lee KY. A novel insertion-induced frameshift mutation of the SLC26A4 gene in a Korean family with Pendred syndrome. Gene. 2012;508(1):135-9. 
5. Busi M, Castiglione A, Taddei Masieri M, Ravani A, Guaran V, Astolfi L, et al. Novel mutations in the SLC26A4 gene. Int J Pediatr Otorhinolaryngol. 2012;76(9):1249-54.

6. Royaux IE, Suzuki K, Mori A, Katoh R, Everett LA, Kohn LD, et al. Pendrin, the protein encoded by the Pendred syndrome gene (PDS), is an apical porter of iodide in the thyroid and is regulated by thyroglobulin in FRTL-5 cells. Endocrinology. 2000;141(2):83945.

7. Gillam MP, Sidhaye AR, Lee EJ, Rutishauser J, Stephan CW, Kopp P. Functional characterization of pendrin in a polarized cell system. Evidence for pendrin-mediated apical iodide efflux. J Biol Chem. 2004;279(13):13004-10.

8. Yoshida A, Hisatome I, Taniguchi S, Sasaki N, Yamamoto Y, Miake $\mathrm{J}$, et al. Mechanism of iodide/chloride exchange by pendrin. Endocrinology. 2004;145(9):4301-8.

9. Kara C, Kılıç M, Uçaktürk A, Aydın M. Congenital goitrous hypothyroidism, deafness and iodide organification defect in four siblings: Pendred or pseudo-Pendred syndrome? J Clin Res Pediatr Endocrinol. 2010;2(2):81-4.

10. Asakura Y, Narumi S, Muroya K, Fujita K, Aida N, Hasagawa T, et al. A patient with Pendred syndrome whose goiter progressed with normal serum thyrotropin and iodine organification. Am J Med Genet A. 2010;152A(7):1793-7.

11. Kopp P. Mutations in the Pendred Syndrome (PDS/SLC26A) gene: an increasingly complex phenotypic spectrum from goiter to thyroid hypoplasia. J Clin Endocrinol Metab. 2014;99(1):67-9.

12. He Z, ZhaoY, Zhang Q, Peng J, Gao J, Sun J, et al. Ultrasonography on thyroid volume of normative neonate. Chin J Med Imaging Technol. 2010; 26(2):396.

13. Szinnai G. Clinical genetics of congenital hypothyroidism. Endocr Dev. 2014;26:60-78.
14. Rastogi MV, LaFranchi SH. Congenital hypothyroidism. Orphanet J Rare Dis. 2010;5:17.

15. Kurtoğlu S, Köroğlu Ş, Baştuğ O, Daar G, Yıkılmaz A, Elmalı F.The comparison of thyroxine versus thyroxine plus oral iodine in the treatment of congenital hypothyroidism due to iodine deficiency. Horm Res Paediatr. 2014;81(6):409-15.

16. Büyükgebiz A. Newborn screening for congenital hypothyroidism. J Pediatr Endocrinol Metab. 2006;19(11):1291-8.

17. LI Q, Su X, Yu J, Zhang S, Liu P, Ji X, et al. Analysis of field survey results for iodine deficiency disorders in high-risk areas of China. Chin J Endemiol. 2009;28(2):197-201.

18. Grasberger H, Refetoff S. Genetic causes of congenital hypothyroidism due to dyshormonogenesis. Curr Opin Pediatr. 2011;23(4):421-8.

19. Ladsous M, Vlaeminck-Guillem V, Dumur V, Vincent C, Dubrulle $F$, Dhaenens CM, et al. Analysis of the thyroid phenotype in 42 patients with Pendred syndrome and nonsyndromic enlargement of the vestibular aqueduct. Thyroid. 2014;24(4):639-48.

20. Reardon W, Coffey R, Phelps PD, Luxon LM, Stephens D, Kendall-Taylor $P$, et al. Pendred syndrome--100 years of underascertainment? QJM. 1997;90(7):443-7.

21. Reardon W, Coffey R, Chowdhury T, Grossman A, Jan H, Britton $\mathrm{K}$, et al. Prevalence, age of onset, and natural history of thyroid disease in Pendred syndrome. J Med Genet. 1999;36(8):595-8.

22. Blons H, Feldmann D, Duval V, Messaz O, Denoyelle F, Loundon $N$, et al. Screening of SLC26A4 (PDS) gene in Pendred's syndrome: a large spectrum of mutations in France and phenotypic heterogeneity. Clin Genet. 2004;66(4):333-40.

23. Kühnen $P$,Turan $S$, Fröhler $S$, Güran T, Abali $S$, Biebermann $H$, et al. Identification of PENDRIN (SLC26A4) mutations in patients with congenital hypothyroidism and "apparent" thyroid dysgenesis. J Clin Endocrinol Metab. 2014;99(1):E169-76. 